\title{
Reduced Cortical Thickness is Associated with the Glutamatergic Regulatory Gene Risk Variant DAOA Arg30Lys in Schizophrenia
}

\author{
C Christoph Schultz*,', Igor Nenadic', Kathrin Koch', Gerd Wagner', Martin Roebel', \\ Claudia Schachtzabel', Thomas W Mühleisen ${ }^{2,3}$, Markus M Nöthen ${ }^{2,3}$, Sven Cichon $^{2,3,4}$, Thomas Deufel ${ }^{5}$, \\ Michael Kiehntopf ${ }^{5}$, Marcella Rietschel ${ }^{6}$, Jürgen R Reichenbach', Heinrich Sauer' and Ralf GM Schlösser ${ }^{1}$ \\ 'Department of Psychiatry and Psychotherapy, Friedrich-Schiller-University Jena, Jena, Germany; ${ }^{2}$ Institute of Human Genetics, University of Bonn, \\ Bonn, Germany; ${ }^{3}$ Department of Genomics, Life and Brain Center, University of Bonn, Bonn, Germany; ${ }^{4}$ Institute of Neuroscience and Medicine \\ (INM-I), Research Center Juelich, Juelich, Germany; ${ }^{5}$ Department of Clinical Chemistry and Laboratory Diagnostics, Friedrich-Schiller-University \\ Jena, Jena, Germany; ${ }^{6}$ Division of Genetic Epidemiology in Psychiatry, Central Institute of Mental Health, Mannheim, Germany; ${ }^{7}$ Medical Physics \\ Group, Institute for Diagnostic and Interventional Radiology, Friedrich-Schiller-University Jena, Jena, Germany
}

\begin{abstract}
In light of current etiological concepts the glutamatergic system plays an essential role for the pathophysiology of the disorder, offering multiple options for new treatment strategies. The D-amino oxidase activator (DAOA) gene is closely connected to the glutamatergic system and its therapeutic and pathophysiological relevance for schizophrenia is therefore intensively debated. In a further step to shed light on the role of DAOA in schizophrenia, we aimed to investigate the association of the functional DAOA Arg30Lys (rs2391 I9I) variant and cortical thickness in schizophrenia. Cortical thickness was computed by an automated surface-based technique (FreeSurfer) in 52 genotyped patients with schizophrenia and 42 healthy controls. Cortical thickness of the entire cortex was compared between risk carriers and non-risk carriers regarding the Arg30Lys polymorphism in patients and healthy controls on the basis of a node-by-node procedure and an automated clustering approach. Risk carriers with schizophrenia show significantly thinner cortex in two almost inversely arranged clusters on the left and right hemisphere comprising middle temporal, inferior parietal, and lateral occipital cortical areas. The clusters encompass an area of $1174 \mathrm{~mm}^{2}$ (left) and $1156 \mathrm{~mm}^{2}$ (right). No significant effect was observed in healthy controls. The finding of our study that the Arg30Lys risk variant is associated with a distinct cortical thinning provides new evidence for the pathophysiological impact of DAOA in schizophrenia. The affected areas are mostly confined to cortical regions with a crucial role in the ToM network and visual processing, which both can be influenced by glutamatergic modulation. Our finding thus underlines the importance of DAOA and related glutamatergic processes as a putative target for therapeutic interventions in schizophrenia.

Neuropsychopharmacology (20II) 36, 1747-1753; doi: I0.1038/npp.201 I.56; published online 20 April 20II
\end{abstract}

Keywords: rs2391191; G72; schizophrenia; cortical thickness; susceptibility gene; DAOA

\section{INTRODUCTION}

In light of current etiological concepts of schizophrenia the glutamatergic system plays an essential role for the pathophysiology of the disorder, offering multiple options for new treatment strategies (Javitt, 2007). Genetic findings of the recent years fundamentally broadened our knowledge

\footnotetext{
*Correspondence: Dr CC Schultz, Department of Psychiatry and Psychotherapy, Friedrich-Schiller-University Jena, Philosophenweg 3, Jena 07740, Germany, Tel: + 493641935665 or + 493641933944 , Fax: + 49364193 5444, E-mail: christoph.schultz@med.uni-jena.de Received I5 November 2010; revised 18 March 2011; accepted 22 March 2011
}

of schizophrenia (O'Donovan et al, 2009) and particularly in view of the glutamatergic system as several identified genes are implicated to interact with the glutamatergic neurotransmission (Coyle, 2006). However, as a further translational step, the identification of the exact pathophysiological mechanisms is mandatory for the development of new treatment strategies. A new emerging approach, imaging genetics, intends to identify the pathophysiological role of genetic variations by analyzing the impact on brain function and structure. In particular, schizophrenia susceptibility genes offering potential targets for new treatment strategies through their biochemical products are of special interest. The G72 gene is one of the identified susceptibility genes in schizophrenia. Its biochemical products play an 
important role in the glutamatergic neurotransmission through interaction with the D-amino acid oxidase (DAAO) and was therefore identified as D-amino oxidase activator (DAOA) (Chumakov et al, 2002). DAOA and related glutamatergic mechanisms are suggested to constitute potential targets for treatment strategies in schizophrenia (Javitt, 2007; Williams, 2009). Genetic variation in this gene was first found to be associated with schizophrenia by Chumakov et al (2002) and Schumacher et al (2004). A recent meta-analysis of all published association studies supported G72 as a risk gene for schizophrenia (DeteraWadleigh and McMahon, 2006b). Furthermore, DAOA variants predicted the progression from prodromal to the first episode in schizophrenia (Mossner et al, 2010). The importance of DAOA gene variants has additionally been demonstrated using functional magnetic resonance imaging (fMRI) (Jansen et al, 2009; Krug et al, 2011). A recent study by Hartz et al (2010) examined the association of a fourlocus haplotype of DAOA, including the rs2391191 SNP, with longitudinal cerebral lobe volume change in schizophrenia. This association was tested between the volumes of the segregated cerebral lobes, total cerebral volume, and the resulting three-allele representations of the DAOA haplotype in a longitudinal design in schizophrenia. A significant association of total frontal lobe volume decrease after a period of on average 3 years of illness for one specific allele representation of the DAOA haplotype was detected, indicating that the DAOA risk variants might be associated with progressive cerebral changes in schizophrenia.

The DAOA rs2391191 (M15) SNP variant showed one of the strongest associations with schizophrenia in a previous meta-analysis (Detera-Wadleigh and McMahon, 2006b). It causes an arginine-to-lysine substitution at codon 30 (Arg30Lys) and might therefore be of putative functional relevance (Donohoe et al, 2007). Recently, it has been shown that the DAOA rs2391191 SNP variant is associated with a significantly lower visual memory capacity in patients with a psychotic disorder (Soronen et al, 2008). Additionally, the rs2391191 SNP variant is associated with impaired verbal memory in schizophrenia (Donohoe et al, 2007). However, the pathophysiological basis for the functional deficits impacted by the rs2391191 SNP remains unclear.

Surface-based MRI studies investigate cortical shape parameters in terms of cortical thickness and curvature. Surface-based MRI studies showed that cortical thickness is reduced in chronic (Kuperberg et al, 2003; Nesvag et al, 2008; Schultz et al, 2010a) and first-episode schizophrenia (Narr et al, 2005; Schultz et al, 2010b) in mainly the frontotemporal, but also parietal regions. ROI analyses showed cortical thinning in key regions of the medial surface, including the anterior cingulum (Fornito et al, 2008) and the entorhinal cortex (Schultz et al, 2010c). As cortical thickness is assumed to reflect neurodevelopmental mechanisms (Hilgetag and Barbas, 2006), cortical thickness might be a suitable parameter for the investigation of a potential association of genetic variants and brain morphology. Thus, cortical thinning might constitute one underlying patho-mechanism of the DAOA Arg30Lys risk variant. However, the association of the DAOA Arg30Lys coding variant and cortical thickness has to our best knowledge not been explored.

\section{Objectives and Hypothesis}

Based on these considerations we compared whole-brain cortical thickness computed by a surface-based MRI method between the different groups in patients and healthy controls according to the genotype status, to identify a potential association of G72 SNP rs2391191 and cortical thickness in patients with schizophrenia and healthy controls. According to the meta-analysis of Detera-Wadleigh and McMahon (2006a), the $\mathrm{A}$ allele represents the risk allele in schizophrenia. Therefore, we hypothesized carriers of the A allele (AA/AG) showing a thinner cortex both in patients with schizophrenia and healthy controls in comparison with homozygous $G$ carriers (GG), reflecting a potential patho-mechanism of this DAOA risk variant.

\section{MATERIALS AND METHODS}

\section{Participants}

We studied 52 patients with schizophrenia and 42 healthy controls. All participants were right-handed (Annett, 1967). Diagnoses were established by a clinical psychiatrist (M Roebel) based on the Structured Clinical Interview for DSM-IV and were confirmed by two independent psychiatrists (RGM Schlösser and CC Schultz). All patients met the DSM-IV criteria for schizophrenia and had no second psychiatric diagnosis. They were on stable medication, mostly with second-generation antipsychotics.

Healthy volunteers were screened for major medical, neurological, and psychiatric history. None of the healthy subjects had a current or history of a psychiatric disorder and first-degree relatives with a psychiatric disorder according to DSM-IV. The exclusion criteria for all participants were neurological disease or damage, medical disorders potentially influencing neurocognitive function. All participants were Caucasian. All participants gave written informed consent to the study approved by the Ethics Committee of the Friedrich-Schiller University. The sociodemographic and psychopathological data are given in Table 1.

\section{MRI Acquisition}

We acquired high-resolution anatomical T1-weighted scans using a 1.5-T Siemens Magnetom Vision whole-body system using a three-dimensional spoiled gradient echo sequence: $1 \mathrm{~mm}$ sagittal slices with $\mathrm{TR}=15 \mathrm{~ms}, \mathrm{TE}=5 \mathrm{~ms}$; flip angle $30^{\circ}$; $\mathrm{FOV}=256$; matrix $=256 \times 256$; number of sagittal slices $=192$.

All scans were inspected for motion artifacts and a neuroradiologist confirmed absence of gross pathological findings.

\section{MR Scan Processing and Calculation of Cortical Thickness}

We used the FreeSurfer software package (version 4.0.5; http://surfer.nmr.mgh.harvard.edu) for processing images (Dale et al, 1999; Fischl et al, 1999). The implemented processing stream comprises removal of non-brain tissue, transformation to Talairach-like space, and segmentation of gray/white matter tissue. White and gray matter boundary 
Table I Demographic and Clinical Data

\begin{tabular}{|c|c|c|c|}
\hline & \multicolumn{2}{|c|}{ Genetic groups } & \multirow[t]{2}{*}{$p$} \\
\hline & GG & AA/AG & \\
\hline \multicolumn{4}{|l|}{ Healthy controls } \\
\hline$n$ & 16 & 26 & \\
\hline Age (years) & $26.1(5.7)$ & $27.9(8.7)$ & 0.74 \\
\hline \multicolumn{4}{|l|}{ Patients } \\
\hline n & 26 & 26 & \\
\hline Age (years) & $28.4(10.3)$ & $30.4(10.4)$ & 0.430 \\
\hline PANSS total & 75.1 (26.5) & $66.9(20.7)$ & 0.63 \\
\hline PANSS pos & $18.2(8.4)$ & $15.9(7.2)$ & 0.76 \\
\hline PANSS neg & $17.5(6.2)$ & |7.| (6.2) & 0.98 \\
\hline Duration of illness (years) & $2.7(3.0)$ & $3.8(6.8)$ & $0.67 \varepsilon$ \\
\hline CPZ equivalents (mg) & $576.5(4 \mid 2.8)$ & $460.9(293.1)$ & 0.40 \\
\hline
\end{tabular}

Abbreviations: CPZ, chlorpromazine; PANSS, positive and negative syndrome scale (Kay et al, 1987). Data expressed as mean (SD). p-values resulting from Mann-Whitney U-tests.

is tessellated and topological defects are automatically corrected. After intensity normalization, transition of gray/white matter and pial boundary is identified by detecting the greatest shift in intensity through surface deformation. The entire cortex of each subject was then visually inspected and any inaccuracies in segmentation were manually edited. After creation of the cortical representations the cerebral cortex is automatically parcellated into anatomical structures (Fischl et al, 2004). Cortical thickness is computed by finding the shortest distance between a given point on the estimated pial surface and the gray/white matter boundary and vice versa, and averaging these two values (Fischl and Dale, 2000). As the generated cortical maps are not restricted to the voxel resolution of the original data, they are capable of detecting submillimeter differences between diagnostic groups. This automated approach of cortical thickness measurement has been validated against manual measurements in schizophrenia (Kuperberg et al, 2003).

\section{Genetic Analyses and Identification of Subgroups}

Fifty-two patients with schizophrenia and 42 healthy controls were genotyped for the G72 SNP (M15= rs2391191). Genotyping was performed as part of a largescale, multi-center study using high-throughput technology including 768 SNPs (GoldenGate assay; Illumina, San Diego) (Treutlein et al, 2009). The primer sequences and PCR conditions can be obtained from the authors upon request. The raw genotypes were quality-controlled by visual inspection of the genotype cluster plots. The 94 participants recruited in the study center Jena underwent structural MRI and were analyzed in the present study. To estimate the replicate error rate for quality comparison purposes, we genotyped a subset of the sample twice. Two out of 94 DNA samples were randomly chosen for this purpose. For the SNPs genotyped in the framework of the multi-center study, all genotypes between duplicates were consistent ( $0 \%$ replicate error rate). In a standard $1 \mathrm{df} \chi^{2}$-test, there were no significant deviations from Hardy-Weinberg equilibrium for the genotype distributions of the studied sample. Based on the considerations given in the section Introduction, we examined only the DAOA Arg30Lys SNP with regard to a potential association with cortical thickness. Depending on the M15 genotype, patients with schizophrenia and healthy controls were divided in subgroups resulting in two genotype subgroups per diagnostic group, ie, $2 \times \mathrm{A} / \mathrm{A}$ and $\mathrm{A} / \mathrm{G} ; 2 \times \mathrm{G} / \mathrm{G}$.

\section{Statistical Analysis}

Statistical cortical maps. Each cortical thickness measurement of each vertex of the subjects' surface was mapped on a common spherical coordinate system using a spherical transformation. The maps were smoothed using a Gaussian kernel of $10 \mathrm{~mm}$. A general linear model controlling for the effect of age was used to estimate differences in cortical thickness between the diagnostic groups according to the genotype in patients and healthy controls separately at each vertex of the surface.

The right and left hemispheres were tested separately.

Monte Carlo simulation and clustering. As a conservative procedure for correction of multiple comparisons, a Monte Carlo simulation with 10000 iterations and cluster analysis was then performed to identify contiguous clusters of significant cortical thickness differences between the genotype groups in patients and healthy controls $(p<0.05)$.

The simulation and clustering approach is implemented in FreeSurfer and is based on the AlphaSim algorithm (Ward, 2000). Significance level is obtained through a combination of a probability threshold and cluster size threshold. The Monte Carlo simulation computes the distribution of the maximum cluster size. The $p$-values of the resulting clusters of the original data expressed as cluster-wise probability (CWP) represent the probability to identify a maximum cluster of that size or larger during the simulation step. Hence, the CWP resulting from the simulation and clustering is equivalent to the overall alpha significance level.

\section{RESULTS}

Schizophrenia patients with the AA/AG genotype in comparison with patients with the GG genotype show a significantly (corrected for multiple comparisons) thinner cortex in two almost inversely arranged clusters on the left and right hemispheres. The clusters comprised parts of the middle temporal, inferior parietal, and lateral occipital cortex of both hemispheres (s. Figure 1). Table 2 summarizes the characteristics of the two clusters.

Among healthy controls the AA/AG carriers showed no significant differences in cortical thickness in comparison with healthy GG carriers.

We additionally performed a genotype $\times$ diagnosis interaction analysis with the same four groups as defined for the comparisons of cortical thickness between the genotype groups (HC: AA/AG; HC: GG; SZ: AA/AG; SZ: GG) and 
applying the same statistical conditions (Monte Carlo simulation, automated clustering). We did not detect a significant difference between the diagnostic groups regarding the effect of the genotypes.

\section{DISCUSSION}

In order to identify a potential patho-mechanism of the glutamatergic regulatory gene variant DAOA Arg30Lys, the present study is the first to specifically investigate the association of the DAOA Arg30Lys variant on cortical morphology in schizophrenia and healthy controls. Our data show that this variant has a significant impact on cortical thickness in schizophrenia but not in healthy controls. In accordance to our hypotheses based on the results of the meta-analysis of the G72 gene (DeteraWadleigh and McMahon, 2006b), A carriers with schizophrenia had a thinner cortex in an area covering the temporo-parietal junction and lateral occipital regions on both hemispheres in comparison with GG carriers.

Although the recent meta-analysis from the SchizophreniaGene Database of Schizophrenia Research Forum (http:// www.schizophreniaforum.org/res/sczgene), which did not

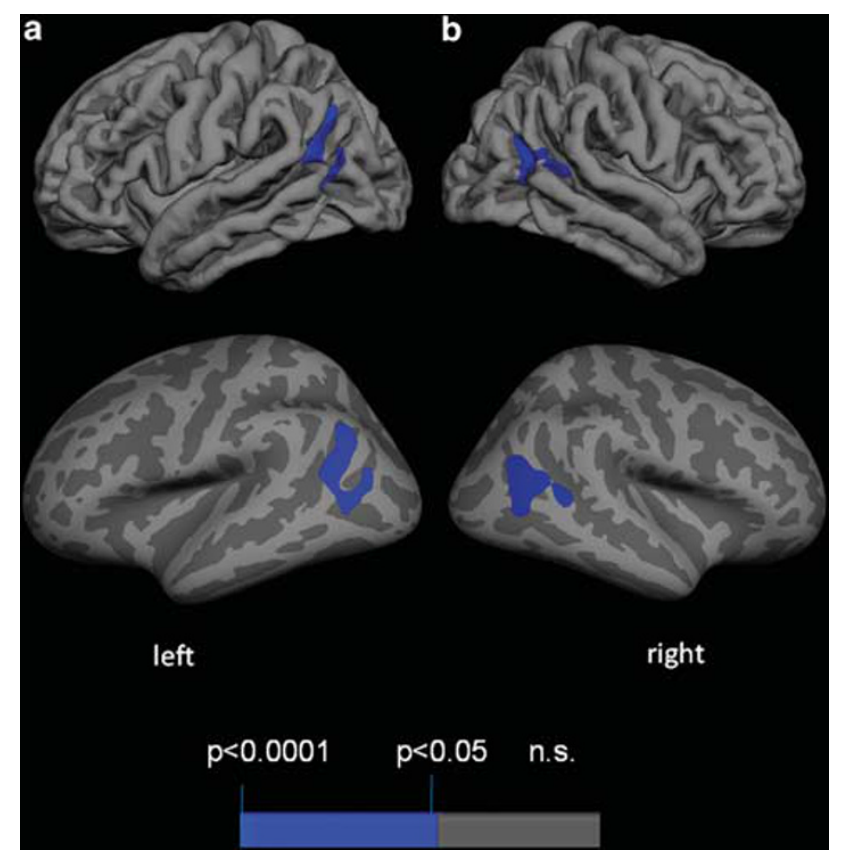

Figure I Cortical statistical maps displaying cortical thickness differences in schizophrenia patients between carriers of AA/AG and GG. Clusters (in blue) indicate significant thinner cortical areas in patients with a AA/AG vs GG genotype (SNP 2391191). Shown is the lateral surface of both hemispheres, pial and inflated view, $p$-values corrected for multiple comparison. include family-based association data, did not indicate a significant association between the Arg30Lys SNP and schizophrenia, our data implicate that the rs2391191 variant has a distinct impact on cerebral morphology in schizophrenia and might influence pathophysiological processes relevant for the disorder.

\section{Reduced Cortical Thickness in Risk Carriers and Potential Functional Implications: the ToM Network and Visual Processing Deficits in Schizophrenia}

Beyond other cortical regions comprising the medial prefrontal (Vollm et al, 2006) and the posterior cingulate cortex (Gobbini et al, 2007), the temporo-parietal junction has been indicated to be an anatomical node of the theory of mind (ToM) network (Andreasen et al, 2008; Brune et al, 2008). Impairments of the ToM network are confirmed by two meta-analyses (Bora et al, 2009; Sprong et al, 2007) and can be regarded as a trait marker for schizophrenia. In the present study cortical thinning of the temporo-parietal junction has been shown in G72 risk allele carriers. Thus, this subtype of schizophrenia patients might be predominantly vulnerable to developing ToM deficits. This assumption would additionally strengthen the view that the phenotypical heterogeneity of schizophrenia might be reduced by defining endophenotypical and genetically subgroups. It should be emphasized, however, that this assumption is very speculative and further, particularly multimodal, studies are needed to elucidate this issue.

In addition to the observed reduced cortical thickness of the temporo-parietal junction, the clusters of cortical thinning in G72 risk carriers comprise the inferior parietal and lateral occipital areas. These cortical regions are mostly confined to parts of the 'dorsal stream' of visual processing in humans (Ettlinger, 1990). Visual processing deficits are core parts of the pathophysiology in schizophrenia (Coleman et al, 2009; Kantrowitz et al, 2009). fMRI studies showed that the lateral occipital areas, the inferior parietal cortex, and the human motion-selective cortex (hMT) show disturbed activation during visual processing (Green et al, 2005). Studies in unaffected siblings of schizophrenia patients illustrated that visual processing deficits are related to genetic liability (Green et al, 2006). Interestingly, it has also been shown that ToM impairments in schizophrenia are associated with deficits of motion perception as an upward consequence of altered visual processing (Kelemen et al, 2005). Visual processing has been shown to be modulated by the glutamatergic system (Javitt, 2009). In accordance, a study by Uhlhaas et al (2007) showed visual perception deficits in ketamine users similar to those deficits found in schizophrenia. The G72 gene investigated in the present study is strongly connected to the glutamatergic system.

Table 2 Significant Clusters for the Left and Right Hemisphere (Cluster Size in $\mathrm{mm}^{2}$ ), $p$-Values from the Monte Carlo Simulation, and Clustering as CWP, Resulting from the Vertex-wise Comparison of Cortical Thickness between the Two Patient Groups

\begin{tabular}{lccrr}
\hline Cortex area & Size in $\mathbf{~ m m}^{\mathbf{2}}$ & Talx & Taly & Talz \\
\hline Left middle temporal, inferior parietal, lateral occipital & 1174.9 & -38.9 & -61.5 & 30.7 \\
Right middle temporal, inferior parietal, lateral occipital & 1156.1 & 37.8 & -58.3 & 0.0108 \\
\hline
\end{tabular}


Functional Role of G72 and its Impact as a Potential Target for Therapeutic Interventions in Schizophrenia

The G72 gene is directly involved in the cerebral glutamatergic neurotransmission. The product of G72 (DAOA) interacts with DAAO, which degrades D-amino acids in the human brain. The activity of DAAO is increased in schizophrenia (Habl et al, 2009; Madeira et al, 2008). These findings would imply a relative cerebral deficit of D-amino acids such as D-serine, which act as modulator at the $N$-methyl-D-aspartate (NMDA) ionotropic glutamate receptor. Indeed, decreased D-Serine serum levels have been shown in schizophrenia (Hashimoto et al, 2003). The D-amino acid oxidase activator (DAOA) might act as a negative effector for the DAAO (Sacchi et al, 2008). Thus, a genetically impaired and inactive DAOA would lead to hyperactivity of DAAO. Subsequently, hyperactivity of DAAO may result in decreased D-serine levels. Decreased D-serine levels induce a hypo-function of the glutamatergic system through under-stimulation of NMDA receptors. Dysfunction of NMDA receptors is a key pathophysiology of schizophrenia (Coyle and Tsai, 2004; Coyle et al, 2003; Javitt, 2002). Accordingly, clinical trials showed that add-on treatment with D-serine has a significant beneficial effect on negative and cognitive symptoms in schizophrenia (Heresco-Levy et al, 2005; Tsai et al, 1998).

In accordance to current neurodevelopmental models of schizophrenia (du Bois and Huang, 2007), the present study underlines the relevance of a glutamatergic regulatory gene variant for cortical thickness in regions relevant for schizophrenia. This corroborates the assumption that glutamatergic dysregulation is associated with cortical thinning processes as an underlying neuropathological feature of schizophrenia.

\section{A Potential Cerebral Mechanism Compensating for the Effect of the G72 Risk Variant in Healthy Controls}

The biological effect of the amino-acid change caused by the Arg30Lys variant can be assumed to be present in both healthy subjects and patients. Hence, we hypothesized finding cortical thickness differences between the genotype groups in both patients with schizophrenia and healthy controls. In the present study we did not find an impact of the risk genotype on cortical thickness in healthy controls.

Various cerebral mechanisms might explain this finding. Cortical thinning originated from different processes of brain development and at differing time points. In the early stages of brain development, cortical thickness is impacted by neuronal migration, formation of cortical layers, and building of cortico-cortical connections (Hilgetag and Barbas, 2005). Processes of net synaptic pruning occurring in the adolescence period (Huttenlocher and Dabholkar, 1997) also have an influence on cortical thickness. Later in life, age-related processes additionally lead to cortical thickness reduction, which might be accelerated in schizophrenia (Hulshoff Pol et al, 2002). At which stage a distinct G72 risk genotype impacts these cortical processes is speculative. However, it gets obvious that at every stage a compensatory mechanism (ie, lower synaptic pruning, preservation of accelerated age-related processes) might lead to unaltered cortical morphology in healthy risk carriers so that the critical point of vulnerability to manifest the disorder is not reached.

\section{Potential Limitations of the Study}

The present study used 1.5-T MRI to explore the association of the Arg30Lys polymorphism and cortical thickness in schizophrenia and healthy controls, showing a significant association of the Arg30Lys risk variant and cortical thinning in schizophrenia. As a higher magnetic field strength might be more sensitive for the detection of structural alterations (Di Perri et al, 2009), we cannot definitely exclude that a significant association of the Arg30Lys variant and cortical thickness in healthy controls might get unveiled with the use of a higher scanner field strength. Thus, further studies are needed to shed more light on this issue.

\section{Concluding Remarks}

The principal finding of our study, that the glutamatergic regulatory gene variant DAOA Arg30Lys is associated with a distinct cortical thinning, shows that this variant has an impact on brain morphology in functional relevant areas in schizophrenia. The affected areas are mostly confined to cortical regions constituting central nodes in the ToM network and higher visual processing. Both processes might be influenced by glutamatergic modulation. Our finding thus adds new evidence for the pathophysiological relevance of DAOA in schizophrenia and might open new perspectives for DAOA and related glutamatergic mechanisms as a putative target for therapeutic interventions.

\section{ACKNOWLEDGEMENTS}

This work was supported by the Bundesministerium für Bildung und Forschung, BMBF Grant 01GW0740 and the Interdisciplinary Center for Clinical Research of the University of Jena (IZKF). The sponsor served no role in the study design; in the collection, analysis, and interpretation of data; in the writing of the report; or in the decision to submit the paper for publication.

\section{DISCLOSURE}

The authors declare that HS received compensation from Otsuka and Lilly in 2008. All of the authors reported no financial, personal, or other relationships with other people or organizations that could inappropriately influence, or be perceived to influence, their work.

\section{REFERENCES}

Andreasen NC, Calarge CA, O'Leary DS (2008). Theory of mind and schizophrenia: a positron emission tomography study of medication-free patients. Schizophr Bull 34: 708-719.

Annett M (1967). The binomial distribution of right, mixed and left handedness. Q J Exp Psychol 19: 327-333.

Bora E, Yucel M, Pantelis C (2009). Theory of mind impairment in schizophrenia: meta-analysis. Schizophr Res 109: 1-9.

Brune M, Lissek S, Fuchs N, Witthaus H, Peters S, Nicolas V et al (2008). An fMRI study of theory of mind in schizophrenic patients with 'passivity' symptoms. Neuropsychologia 46: 1992-2001. 
Chumakov I, Blumenfeld M, Guerassimenko O, Cavarec L, Palicio M, Abderrahim $\mathrm{H}$ et al (2002). Genetic and physiological data implicating the new human gene G72 and the gene for D-amino acid oxidase in schizophrenia. Proc Natl Acad Sci USA 99: 13675-13680.

Coleman MJ, Cestnick L, Krastoshevsky O, Krause V, Huang Z, Mendell NR et al (2009). Schizophrenia patients show deficits in shifts of attention to different levels of global-local stimuli: evidence for magnocellular dysfunction. Schizophr Bull 35: 1108-1116.

Coyle JT (2006). Glutamate and schizophrenia: beyond the dopamine hypothesis. Cell Mol Neurobiol 26: 365-384.

Coyle JT, Tsai G (2004). NMDA receptor function, neuroplasticity, and the pathophysiology of schizophrenia. Int Rev Neurobiol 59: 491-515.

Coyle JT, Tsai G, Goff D (2003). Converging evidence of NMDA receptor hypofunction in the pathophysiology of schizophrenia. Ann NY Acad Sci 1003: 318-327.

Dale AM, Fischl B, Sereno MI (1999). Cortical surface-based analysis. I. Segmentation and surface reconstruction. Neuroimage 9: 179-194.

Detera-Wadleigh S, McMahon F (2006a). G72/G30 in schizophrenia and bipolar disorder: review and meta-analysis. Biol Psychiatry 60: 106-114.

Detera-Wadleigh SD, McMahon FJ (2006b). G72/G30 in schizophrenia and bipolar disorder: review and meta-analysis. Biol Psychiatry 60: 106-114.

Di Perri C, Dwyer MG, Wack DS, Cox JL, Hashmi K, Saluste E et al (2009). Signal abnormalities on 1.5 and 3 Tesla brain MRI in multiple sclerosis patients and healthy controls. A morphological and spatial quantitative comparison study. Neuroimage 47: 1352-1362.

Donohoe G, Morris DW, Robertson IH, McGhee KA, Murphy K, Kenny $\mathrm{N}$ et al (2007). DAOA ARG30LYS and verbal memory function in schizophrenia. Mol Psychiatry 12: 795-796.

du Bois TM, Huang XF (2007). Early brain development disruption from NMDA receptor hypofunction: relevance to schizophrenia. Brain Res Rev 53: 260-270.

Ettlinger G (1990). 'Object vision' and 'spatial vision': the neuropsychological evidence for the distinction. Cortex 26: 319-341.

Fischl B, Dale AM (2000). Measuring the thickness of the human cerebral cortex from magnetic resonance images. Proc Natl Acad Sci USA 97: 11050-11055.

Fischl B, Sereno MI, Dale AM (1999). Cortical surface-based analysis. II: inflation, flattening, and a surface-based coordinate system. Neuroimage 9: 195-207.

Fischl B, van der Kouwe A, Destrieux C, Halgren E, Segonne F, Salat DH et al (2004). Automatically parcellating the human cerebral cortex. Cereb Cortex 14: 11-22.

Fornito A, Yucel M, Wood SJ, Adamson C, Velakoulis D, Saling MM et al (2008). Surface-based morphometry of the anterior cingulate cortex in first episode schizophrenia. Hum Brain Mapp 29: 478-489.

Gobbini MI, Koralek AC, Bryan RE, Montgomery KJ, Haxby JV (2007). Two takes on the social brain: a comparison of theory of mind tasks. J Cogn Neurosci 19: 1803-1814.

Green MF, Glahn D, Engel SA, Nuechterlein KH, Sabb F, Strojwas $M$ et al (2005). Regional brain activity associated with visual backward masking. J Cogn Neurosci 17: 13-23.

Green MF, Nuechterlein KH, Breitmeyer B, Mintz J (2006). Forward and backward visual masking in unaffected siblings of schizophrenic patients. Biol Psychiatry 59: 446-451.

Habl G, Zink M, Petroianu G, Bauer M, Schneider-Axmann T, von Wilmsdorff $\mathrm{M}$ et al (2009). Increased D-amino acid oxidase expression in the bilateral hippocampal CA4 of schizophrenic patients: a post-mortem study. J Neural Transm 116: 1657-1665. Hartz SM, Ho BC, Andreasen NC, Librant A, Rudd D, Epping EA et al (2010). G72 influences longitudinal change in frontal lobe volume in schizophrenia. Am J Med Genet B Neuropsychiatr Genet 153B: 640-647.

Hashimoto K, Fukushima T, Shimizu E, Komatsu N, Watanabe H, Shinoda N et al (2003). Decreased serum levels of D-serine in patients with schizophrenia: evidence in support of the $\mathrm{N}$-methyl-D-aspartate receptor hypofunction hypothesis of schizophrenia. Arch Gen Psychiatry 60: 572-576.

Heresco-Levy U, Javitt DC, Ebstein R, Vass A, Lichtenberg P, Bar G et al (2005). D-serine efficacy as add-on pharmacotherapy to risperidone and olanzapine for treatment-refractory schizophrenia. Biol Psychiatry 57: 577-585.

Hilgetag CC, Barbas H (2005). Developmental mechanics of the primate cerebral cortex. Anat Embryol (Berl) 210: 411-417.

Hilgetag CC, Barbas H (2006). Role of mechanical factors in the morphology of the primate cerebral cortex. PLoS Comput Biol 2: e22.

Hulshoff Pol HE, Schnack HG, Bertens MG, van Haren NE, van der Tweel I, Staal WG et al (2002). Volume changes in gray matter in patients with schizophrenia. Am J Psychiatry 159: 244-250.

Huttenlocher PR, Dabholkar AS (1997). Regional differences in synaptogenesis in human cerebral cortex. J Comp Neurol 387: 167-178.

Jansen A, Krach S, Krug A, Markov V, Eggermann T, Zerres $\mathrm{K}$ et al (2009). A putative high risk diplotype of the G72 gene is in healthy individuals associated with better performance in working memory functions and altered brain activity in the medial temporal lobe. Neuroimage 45: 1002-1008.

Javitt DC (2002). Glycine modulators in schizophrenia. Curr Opin Investig Drugs 3: 1067-1072.

Javitt DC (2007). Glutamate and schizophrenia: phencyclidine, $\mathrm{N}$-methyl-D-aspartate receptors, and dopamine-glutamate interactions. Int Rev Neurobiol 78: 69-108.

Javitt DC (2009). When doors of perception close: bottom-up models of disrupted cognition in schizophrenia. Annu Rev Clin Psychol 5: 249-275.

Kantrowitz JT, Butler PD, Schecter I, Silipo G, Javitt DC (2009). Seeing the world dimly: the impact of early visual deficits on visual experience in schizophrenia. Schizophr Bull 35: 1085-1094.

Kay SR, Fiszbein A, Opler LA (1987). The positive and negative syndrome scale (PANSS) for schizophrenia. Schizophr Bull 13: 261-276.

Kelemen O, Erdelyi R, Pataki I, Benedek G, Janka Z, Keri S (2005). Theory of mind and motion perception in schizophrenia. Neuropsychology 19: 494-500.

Krug A, Markov V, Krach S, Jansen A, Zerres K, Eggermann T et al (2011). Genetic variation in G72 correlates with brain activation in the right middle temporal gyrus in a verbal fluency task in healthy individuals. Hum Brain Mapp 32: 118-126.

Kuperberg GR, Broome MR, McGuire PK, David AS, Eddy M, Ozawa F et al (2003). Regionally localized thinning of the cerebral cortex in schizophrenia. Arch Gen Psychiatry 60: 878-888.

Madeira C, Freitas ME, Vargas-Lopes C, Wolosker H, Panizzutti R (2008). Increased brain D-amino acid oxidase (DAAO) activity in schizophrenia. Schizophr Res 101: 76-83.

Mossner R, Schuhmacher A, Wagner M, Quednow BB, Frommann I, Kuhn KU et al (2010). DAOA/G72 predicts the progression of prodromal syndromes to first episode psychosis. Eur Arch Psychiatry Clin Neurosci 260: 209-215.

Narr KL, Bilder RM, Toga AW, Woods RP, Rex DE, Szeszko PR et al (2005). Mapping cortical thickness and gray matter concentration in first episode schizophrenia. Cereb Cortex 15: 708-719.

Nesvag R, Lawyer G, Varnas K, Fjell AM, Walhovd KB, Frigessi A et al (2008). Regional thinning of the cerebral cortex in schizophrenia: effects of diagnosis, age and antipsychotic medication. Schizophr Res 98: 16-28.

O'Donovan M, Craddock N, Owen M (2009). Genetics of psychosis; insights from views across the genome. Hum Genet 126: 3-12. 
Sacchi S, Bernasconi M, Martineau M, Mothet JP, Ruzzene M, Pilone MS et al (2008). pLG72 modulates intracellular D-serine levels through its interaction with $\mathrm{D}$-amino acid oxidase: effect on schizophrenia susceptibility. J Biol Chem 283: 22244-22256.

Schultz CC, Koch K, Wagner G, Roebel M, Nenadic I, Schachtzabel $\mathrm{C}$ et al (2010a). Complex pattern of cortical thinning in schizophrenia: results from an automated surface based analysis of cortical thickness. Psychiatry Res 182: 134-140.

Schultz CC, Koch K, Wagner G, Roebel M, Schachtzabel C, Gaser C et al (2010b). Reduced cortical thickness in first episode schizophrenia. Schizophr Res 116: 204-209.

Schultz CC, Koch K, Wagner G, Roebel M, Schachtzabel C, Nenadic I et al (2010c). Psychopathological correlates of the entorhinal cortical shape in schizophrenia. Eur Arch Psychiatry Clin Neurosci 260: 351-358.

Schumacher J, Jamra RA, Freudenberg J, Becker T, Ohlraun S, Otte AC et al (2004). Examination of G72 and D-amino-acid oxidase as genetic risk factors for schizophrenia and bipolar affective disorder. Mol Psychiatry 9: 203-207.

Soronen P, Silander K, Antila M, Palo OM, Tuulio-Henriksson A, Kieseppa $\mathrm{T}$ et al (2008). Association of a nonsynonymous variant of DAOA with visuospatial ability in a bipolar family sample. Biol Psychiatry 64: 438-442.
Sprong M, Schothorst P, Vos E, Hox J, van Engeland H (2007). Theory of mind in schizophrenia: meta-analysis. Br J Psychiatry 191: 5-13. Treutlein J, Muhleisen TW, Frank J, Mattheisen M, Herms S, Ludwig KU et al (2009). Dissection of phenotype reveals possible association between schizophrenia and glutamate receptor delta 1 (GRID1) gene promoter. Schizophr Res 111: 123-130.

Tsai G, Yang P, Chung LC, Lange N, Coyle JT (1998). D-serine added to antipsychotics for the treatment of schizophrenia. Biol Psychiatry 44: 1081-1089.

Uhlhaas PJ, Millard I, Muetzelfeldt L, Curran HV, Morgan CJ (2007). Perceptual organization in ketamine users: preliminary evidence of deficits on night of drug use but not 3 days later. J Psychopharmacol 21: 347-352.

Vollm BA, Taylor AN, Richardson P, Corcoran R, Stirling J, McKie $S$ et al (2006). Neuronal correlates of theory of mind and empathy: a functional magnetic resonance imaging study in a nonverbal task. Neuroimage 29: 90-98.

Ward BD (2000). Simultaneous inference for FMRI data. AFNI 3dDeconvolve Documentation,. Medical College of Wisconsin: Milwaukee, Wisconsin.

Williams M (2009). Commentary: genome-based CNS drug discovery: D-amino acid oxidase (DAAO) as a novel target for antipsychotic medications: progress and challenges. Biochem Pharmacol 78: 1360-1365. 